\title{
Effect of Liposome Surface Charge and Peptide Side Chain Charge Density on Antimicrobial Peptide-Membrane Binding as Determined by Circular Dichroism and Nuclear Magnetic Resonance Spectroscopy
}

\author{
Takafumi Urushibara and Rickey Hicks*
}

Department of Chemistry, East Carolina University, Science and Technology Building, Greenville, North Carolina, USA

\begin{abstract}
In this investigation the effect of varying the surface charge and hydrophobicity of liposomes of mixed phospholipid compositions on the binding of four of the AMPs was determined using Circular Dichroism Spectroscopy, ${ }^{1} \mathrm{H}$ NMR and Diffusion Ordered Spectroscopy NMR methods. The following mixed anionic liposomes: 3:1 POPC/POPG, 4:1 POPC/POPG, 5:1 POPC/POPG, 3:1 POPC/POPS, 4:1 POPC/POPS, 5:1 POPC/POPS, and the following mixed zwitter ionic liposomes: 3:1 POPC/POPE, 4:1 POPC/POPE and 5:1 POPC/POPE were used in this investigation. The results obtained from the mixed anionic liposomes study indicated even when the net change on the liposome are the same, local differences in charge density and hydrophobicity will result in different physicochemical surface properties that will dramatically affect the mechanism of AMP binding. The results obtained from the mixed zwitterionic liposomes study indicated that very small changes in the charged amine going from an ammonium salt to a quaternary amine salt will result in different physicochemical surface properties that can inhibit or dramatically reduce AMP binding. This observation is particularly important when designing physicochemical investigations of model membrane of Gram Negative bacteria.
\end{abstract}

\begin{abstract}
Abbreviations: AMP: Antimicrobial Peptide; CD : Circular Dichroism; Dab: Diaminobutionic Acid; DOSY: Diffusion Ordered Spectroscopy; DPC: Dodecylphosphocholine; Dpr: Diaminopropionic acid; ITC: isothermal titration calorimetry; LUV: Large Unilamellar Vesicles; MIC: Minimum Inhibitory Concentration; NMR: Nuclear Magnetic Resonance; Oic: Octahydroindolecarboxylic Acid; POPC: 1-Palmitoyl-2-Oleoyl-sn-Glycero-3-Phosphocholine; POPE: L- $\alpha$-phosphatidylethanolamine; POPG: 1-Palmitoyl-2-Oleoyl-snGlycero-3-[Phospho-rac-(1-glycerol)] (Sodium Salt); POPS: L-aphosphatidylserine; QSAR: Quantitative Structure Activity Relationship; Tic : Tetra hydro isoquinoline carboxylic acid; SDS: Sodium Dodecyl Sulfate; SUV: Small Unilamellar Vesicles
\end{abstract}

\section{Introduction}

Antimicrobial peptides (AMP) generally exhibit antibiotic activity by some type of membrane disruption and have shown promise in preclinical and in clinical studies as potential therapeutic agents [1-6]. AMP's are generally small highly positively charged [7] amphipathic peptides $[4,8,9]$. They have evolved in almost every class of living organism as a host defense mechanism against invading microorganisms including bacteria, fungi, protozoa and parasites [10-14].

Because of the effect of the amphipathic nature of AMPs on their surface physicochemical properties, it has been proposed that the inherent selectivity exhibited by AMPs for prokaryotic vs eukaryotic cells is derived from the differences in the interactions that occur between the AMP and the surface physicochemical properties that result from the variations in the phospholipid compositions of their respective membranes [13,15]. Eukaryotic membranes contain a much higher concentration of zwitter ionic phospholipids, while the membranes of prokaryotic organisms, such as bacteria, contain a high percentage of negatively charged phospholipids [5,14,16-20]. The electrostatic interactions that occur between an AMP and the surface of a phospholipid membrane are believed to be the dominant physicochemical property associated with organism selectivity [2123]. Hancock and co-workers [24] have extended this hypothesis to propose the differences in membrane composition between different strains of bacteria are responsible for the diversity in the potency and selectivity exhibited by a particular AMP against different strains of bacteria. For Gram positive strains such as Staphylococcus aureus, $57 \%$ of the phospholipids composition of the membrane is the anionic lipid 1-Palmitoyl-2-Oleoyl-sn-Glycero-3-[Phospho-rac-(1-glycerol)] (POPG), while for Staphylococcus epidermidis, the percentage of POPG increases to $90 \%$, and for Bacillus subtilus the percentage of POPG is only $29 \%$ [25]. The membranes of the Gram negative strains Salmonella typimurium, Pesudomonascepacia and Escherichia coli contain only 33, 18 and 6 percent POPG respectively [25], while the membranes of Klebsiella pneumoniae and Pseudomonas aeruginosa contain 5 and 21 percent POPG respectively [26]. The membranes of many Gramnegative bacteria contain high concentrations of the zwitterionic lipid 1-Palmitoyl-2-Oleoyl-sn-Glycero-3-Phosphoethanolamine (POPE) instead of POPC, for example Escherichia coli, Klebsiella pneumonia and Pseudomonas aeruginosa are 80, 82 and 60 percent POPE respectively [26].

In our laboratory we developed a series of novel AMPs by incorporating various unnatural amino acids into the primary amino acid sequence with the intent to introduce specific physicochemical properties to control membrane binding [27]. These AMPs exhibited in vitro minimum inhibitory concentrations (MIC) in the low $\mu \mathrm{M}$

\footnotetext{
*Corresponding author: Rickey Hicks, Department of Chemistry, East Carolina University, Suite 300, Science and Technology, Greenville, North Carolina, USA, Tel: 252-328-9702; Fax: 252-328-6210; E-mail: hicksr@ecu.edu

Received August 26, 2013; Accepted September 25, 2013; Published September 27, 2013

Citation: Urushibara T, Hicks R (2013) Effect of Liposome Surface Charge and Peptide Side Chain Charge Density on Antimicrobial Peptide-Membrane Binding as Determined by Circular Dichroism and Nuclear Magnetic Resonance Spectroscopy. J Membra Sci Technol 3: 124. doi:10.4172/2155-9589.1000124

Copyright: (c) 2013 Urushibara T, et al. This is an open-access article distributed under the terms of the Creative Commons Attribution License, which permits unrestricted use, distribution, and reproduction in any medium, provided the original author and source are credited.
} 
Citation: Urushibara T, Hicks R (2013) Effect of Liposome Surface Charge and Peptide Side Chain Charge Density on Antimicrobial PeptideMembrane Binding as Determined by Circular Dichroism and Nuclear Magnetic Resonance Spectroscopy. J Membra Sci Technol 3: 124. doi:10.4172/2155-9589.1000124

to high $\mathrm{nM}$ range against a variety of bacteria [27,28], including drug resistant strains of Enterococcus faecium, Staphylococcus aureus, Klebsiellapnemoniae, Acinetobacterbaumannii, Pseudomonas aeruginosa, and Enterobacter species [29].

The intent of this investigation is to determine the effect of varying the surface charge and hydrophobicity of liposomes of mixed phospholipid compositions on the binding of four of the AMPs developed in our laboratory in order to shed light on the physicochemical requirements for membrane selectivity. The peptide structure of the AMPs in this study incorporated three dipeptide units consisting of the conformationally restrained unnatural amino acids Tic (Tetrahydroisoquinolinecarboxylic acid)-Oic (Octahydroindolecarboxylic acid) into the peptide's primary sequence, Ac-GF-Tic-Oic-GX-Tic-Oic-GF-Tic-Oic-GX-Tic-XXXX$\mathrm{CONH}_{2}$, where $\mathbf{X}$ may be one of the following basic amino acids, Lys, Orn, Dab, Dpr or Arg [27]. The Tic-Oic dipeptide units induce local regions of reduced molecular flexibility onto the peptide backbone and thus result in a reduction of the total conformational flexibility of the peptide during lipid binding. The length of the side chains of the basic residues varies from one to four carbons atoms and defines the distance between the polypeptide backbone and the positively charged side chain nitrogen. This distance is involved in determining the overall surface charge density as well as defining the distance between the membrane surface and the polypeptide backbone. The amino acid sequences of the AMPs used in this investigation are given in Table $1[27,28]$.

\section{Methods}

Monobasic and dibasic sodium phosphate was purchased from Fischer Scientific. L- $\alpha$-phosphatidyl choline, L- $\alpha$ phosphatidylethanolamine, L- $\alpha$-phosphatidylcholine -sodium salt and L- $\alpha$-phosphatidylserine were purchased from Avanti Polar Lipids. All chemicals were used without further purification.

\section{Peptide synthesis}

The AMPs used in these investigation were synthesized either manually using tBOC chemistry or with an automated peptide synthesizer using FMOC chemistry $[30,31]$ as previously reported $[27,28,32]$.

\section{Preparation of mixed POPC/POPE, POPC/POPS and POPC/ POPG SUVs}

The appropriate amount of dry 3:1 POPC/POPG, 4:1 POPC/POPG, 5:1 POPC/POPG, 3:1 POPC/POPS, 4:1 POPC/POPS, 5:1 POPC/ POPS, 3:1 POPC/POPE, 4:1 POPC/POPE and 5:1 POPC/POPE(mol to mol) was weighed to yield a final lipid concentration of $35 \mathrm{mM}$. The lipid was hydrated with $2 \mathrm{~mL}$ of buffer ( $40 \mathrm{mM}$ sodium phosphate, $\mathrm{pH}=6.8$ ) and vortexed extensively. SUVs were prepared by sonication of the milky lipid suspension using a titanium tip ultra-sonicator (QsonicaSonicators model Q55) for approximately 40 minutes in an ice bath until the solution became transparent. The titanium debris were removed by centrifugation at $8,800 \mathrm{rev} / \mathrm{min}$ for 10 minutes using an Eppendorf table top centrifuge [33]. Final lipid concentration used for CD studies was $3.4 \mathrm{mM}$.

\begin{tabular}{|c|l|}
\hline $\begin{aligned} \text { Comp \# } \\
\text { Amino Acid Sequence }\end{aligned}$ & Ac-GF-Tic-Oic-GK-Tic-Oic-GF-Tic-Oic-GK-Tic-KKKK-CONH \\
\hline $\mathbf{4 3}$ & $\begin{array}{l}\text { Ac-GF-Tic-Oic-G-Orn-Tic-Oic-GF-Tic-Oic-G-Orn-Tic-Orn-Orn-Orn-Orn- } \\
\text { CONH }\end{array}$ \\
\hline $\mathbf{5 3}$ & $\begin{array}{l}\text { Ac-GF-Tic-Oic-G-Dab-Tic-Oic-GF-Tic-Oic-G-Dab-Tic-Dab-Dab-Dab- } \\
\text { Dab-CONH }\end{array}$ \\
\hline $\mathbf{5 6}$ & Ac-GF-Tic-Oic-GR-Tic-Oic-GF-Tic-Oic-GR-Tic-RRRR-CONH $_{2}$ \\
\hline
\end{tabular}

Table 1: Amino Acid Sequences of the Peptides used in this Investigation.

\section{Circular dichroism}

CD spectroscopy is very sensitive and its use to monitor conformational changes in peptides and proteins has been well documented [34-36]. Traditionally, SUVs have been employed almost exclusively to investigate the binding of peptides and proteins with lipids in CD studies in order to minimize the contribution of light scattering on the spectra observed with LUVs [34,37]. Peptide concentrations used in this investigation ranged between 150-200 $\mu \mathrm{M}$. All CD spectra were obtained by acquiring 8 scans on a JASCO J-815 CD Spectrometer using a $0.1 \mathrm{~mm}$ cylindrical quartz cell (Starna Cells, Atascadero, CA) from 260 to $195 \mathrm{~nm}$ at $20 \mathrm{~nm} / \mathrm{min}, 1 \mathrm{~nm}$ bandwidth, data pitch $0.2 \mathrm{~nm}$, response time $2.0 \mathrm{sec}$ and 5 mdeg sensitivity at room temperature $\left(\sim 25^{\circ} \mathrm{C}\right)$. Contributions due to the mixed SUVs were eliminated by subtracting the lipid spectra of the corresponding peptide-free solutions. All analysis of CD spectra will be conducted after smoothing (with a means-movement function) using the JASCO Spectra Analysis program [32,38,39]. CD spectra that exhibited HT values of greater than 400 were not used due to excessive light scattering and / or absorption.

\section{NMR experiments}

Samples were prepared by dissolving the peptides $(0.7$ to $1.7 \mathrm{mM})$ in $80 \mathrm{mMperdeuterated} \mathrm{sodium} \mathrm{acetate} \mathrm{buffer} \mathrm{at} \mathrm{a} \mathrm{pH}$ of 6.80 in $\mathrm{D}_{2} \mathrm{O}$, with the appropriate mixed liposome (4:1 POPC/POPE, 4:1 POPC/POPG, and 4:1 POPC/POPS) in the same buffer. All ${ }^{1} \mathrm{H}$ spectra were collected on a Bruker Avance III $400 \mathrm{MHz}$ NMR spectrometer equipped with a 5 $\mathrm{mm}$ direct observe broad-band probe with a Z-gradient. All spectra were collected at a temperature of $298 \mathrm{~K}$. Data was collected with a spectral width of $4,000 \mathrm{~Hz}$, using $64 \mathrm{~K}$ data points, and 64 fids were collected per experiment. Data was processed using exponential multiplication with a line-broadening function of $1.0 \mathrm{~Hz}$. All DOSY experiments were conducted using the bipolar phase longitudinal encode decode (BPPLED) pulse sequence developed by Johnson and coworkers [40]. The bipolar pulse pair consists of two gradient pulses of equal strength and duration in opposite phase to each other. A 180 degree rf pulse is located at midpoint between the two bipolar pulse pairs [40]. The advantage of the bipolar phase pulse $(3 \mu \mathrm{s})$ is that it is self-compensating which removes any imperfections in the pulse sequence [40]. All DOSY experiments were conducted on a Bruker Avance III $400 \mathrm{MHz}$ NMR spectrometer equipped with a $5 \mathrm{~mm}$ direct observe broad-band probe with a Z-gradient. All spectra were collected at a temperature of $298 \mathrm{~K}$. Data was collected with a spectral width of $4,000 \mathrm{~Hz}$, using $64 \mathrm{~K}$ data points, and 32 fids were collected per experiment. The NMR signal obtained from the BPP-LED sequence is attenuated by increasing the strength of the applied gradient pulses in 16 steps from $2 \%$ to $95 \%$. The gradient strength for the probe used was 42 gauss $/ \mathrm{cm}$. The diffusion delay period of $150 \mathrm{~ms}$ was used. Data was processed using exponential multiplication with a line-broadening function of $5 \mathrm{~Hz}$. The intensity of the observed NMR in the DOSY signal is related to the apparent diffusion coefficient by equation 1 [40-42].

$$
I_{i}=I_{0 i} \exp \left[-D_{i} \gamma^{2} \delta^{2} \gamma^{2}(\Delta-\delta / 3)\right]
$$

Where $I_{i}$ is the intensity of the $i^{\text {th }}$ signal in the presence of the gradient pulse, $I_{0 i}$ is the intensity of the $i$ th signal in the absence of the gradient pulse, $\gamma$ is the magneto gyric ratio of the observed nucleus (in this case proton) $\delta$ and $g$ are the gradient pulse duration and strength respectively, $\Delta$ is the time between the bipolar pulse pairs (often referred to as the diffusion time) and $D_{i}$ is the diffusion coefficient of the $i$ th species [40-42]. The program "dosy2d" provided by Bruker Instruments in Topspin version 3.0 was used to fit the NMR data and to obtain the 
Citation: Urushibara T, Hicks R (2013) Effect of Liposome Surface Charge and Peptide Side Chain Charge Density on Antimicrobial PeptideMembrane Binding as Determined by Circular Dichroism and Nuclear Magnetic Resonance Spectroscopy. J Membra Sci Technol 3: 124. doi:10.4172/2155-9589.1000124

Page 3 of 9

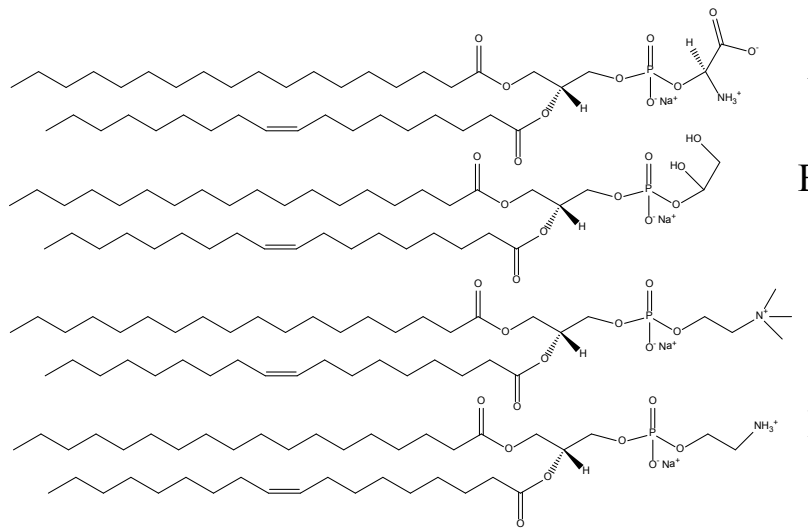

A.

B.

C.

Figure 1: Structure of the phospholipids used in this study. A) POPS, B) POPG, C) POPC, D) POPE.

\begin{tabular}{|c|c|c|}
\hline Amino Acid Residue $^{-}$ & Distance $^{\mathbf{a}}$ & ${\text { Hydrophobicity } \text { value }^{\mathbf{b}}}$ \\
\hline Dab & $3.9 \AA$ & -9.5 \\
\hline Orn & $5.0 \AA$ & -9.0 \\
\hline Lys & $6.4 \AA$ & -9.9 \\
\hline Arg & $7.0 \AA$ & -10.0 \\
\hline
\end{tabular}

a. distance in angstroms from $\mathrm{C}^{\alpha}$ carbon of the peptide backbone to the positively charged nitrogen atom(s)

b. combined consensus scale hydrophobicity for each charge residue

Table 2: Physical Data for the basic amino acid residues used in this study.

diffusion coefficients. The diffusion coefficients were reported to two decimal places with error bars of \pm 0.075 or an error range of $0.15 \mathrm{~m}^{2} / \mathrm{s}$.

\section{Results and Discussion}

The intent of this investigation as previously stated is to determine the effect of varying the surface charge and hydrophobicity of liposomes of mixed phospholipid compositions on the binding of four of the AMPs in order to shed light on the physicochemical requirements for membrane selectivity. We have previously reported that these AMPs exhibited different CD spectra in the presence of zwitter ionic DPC and anionic SDS micelles as well as very different spectra in the presence of zwitter ionic POPC and anionic 4:1 POPC/POPG SUVs indicating that they adopt different conformations on interaction with the zwitterionic and anionic membrane models. Adopting different conformations on binding to zwiterionic and anionic membrane models is a requirement for organism selectivity. This is because changing the conformation of an AMP changes its amphipathic nature which in turn changes its physicochemical surface properties $[27,43]$. The changes in the conformation/physicochemical properties of the AMP will lead to different mechanisms of interaction with zwitter ionic and anionic liposomes and thus to organism selectivity [32,44-46]. This observation was supported by ITC and calcein leakage data that indicated that these AMPs interact via very different mechanisms with anionic and zwitterionic LUVs [32,44-46]. The enthalpy for the binding of these AMPs to pure POPC LUVs directly correlates to the length of side chain of the basic amino acid residues. The enthalpy of binding of these AMPs to 4:1 POPC/POPG, however did not [46]. The AMP containing the Dpr residues (the shortest length spacer) interacts very differently with both POPC and 4:1 POPC/POPG LUVs compared to the other four compounds [46]. At this time we are unable to provide an explanation for this; so for this reason the AMP containing the Dpr residues was not included in this study.
Four different phospholipids, two zwitterionic lipids; L- $\alpha$ phosphatidylcholine (POPC) (one negatively charged and one positively charged functional groups-net charge $=0$ ), $\mathrm{L}$ - $\alpha$ phosphatidylethanolamine (POPE) (one negatively charged and one positively charged functional group-net charge $=0$ ) and two anionic lipids; L- $\alpha$-phosphatidylcholine -sodium salt (POPG) (one negatively charged functional group-net charge $=-1), \quad L-\alpha-$ phosphatidylserine (POPS) (two negatively charged and one positively charged functional groups-net charge $=-1$ ) were selected for this study due to their differing physicochemical surface properties. For example even though the net charge for both POPG and POPS is -1, the local electrostatic surface charges are different. POPG contains one negatively charged group, while POPS contains a negatively charged group as well as a zwitter ionic serine amino acid residue. The surface charge of a membrane is the result of the cumulative effect of all of the charges on the polar head groups contained in the membrane [47]. Mixed SUV liposomes containing either; POPC/POPE, POPC/POPG or POPC/POPS in molar ratios of 3:1, 4:1 and 5:1 were prepared using standard methods [33]. The structures of these four phospholipids are given in Figure 1.

Analysis of in vitro biological data indicates that both the hydrophobicity and the charge distribution of side chain of the basic amino acid residues; contribute to defining antibacterial activity [48].

Compound 23 incorporates Lys residues which contain a side chain of four carbon atoms; compound 43 incorporates Orn residues which contain a side chain of three carbon atoms, while compound 53 incorporates Dab (diamino butanic acid) residues which contain a side chain of two carbon atoms. Changing the number of carbon atoms in the side chain of the basic amino acids changes the overall hydrophobicity of the residue. The combined Consensus Scale (CCS) (hydrophobicity) developed by Tossi and co-workers [49], for each residue used is given in Table 2. Compound 56, contains a side chain guanidinium group where the positive charge is delocalized over two nitrogen atoms instead of only one nitrogen atom as is the case with the other three amine analogs. The shortening of the length of the charged side chains will result in less side chain flexibility and bring the side chain positive charge closer to the electronegative oxygen of carbonyl

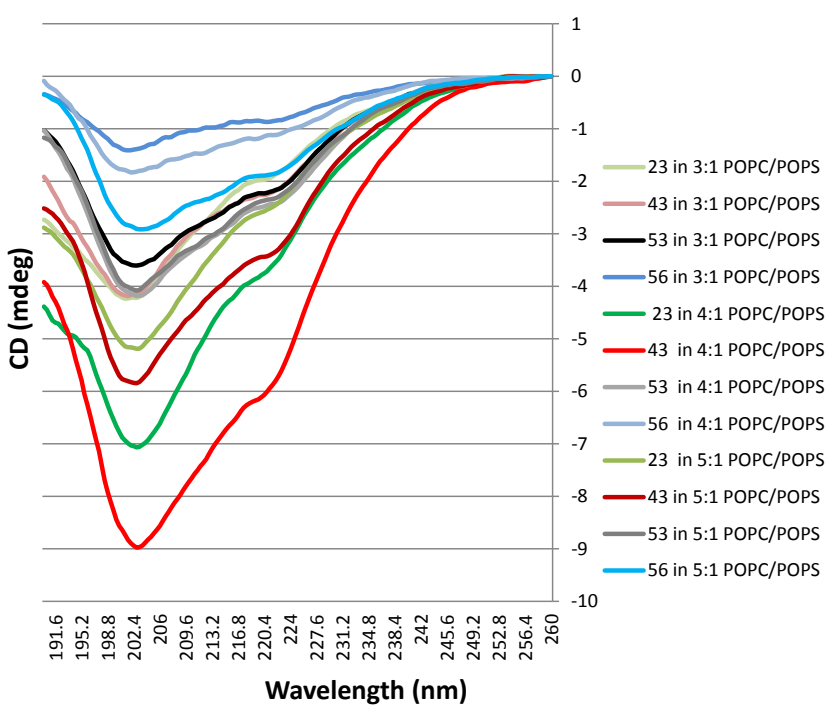

Figure 2: CD spectra of compounds 23 (green shades) 43 (red shades) 53 (black/gray shades) and compound 56 (blue shades) in the presence of $3: 1$ POPC/POPS SUVs, 4:1 POPC/POPS SUVs, and 5:1 POPC/POPS SUVs. 
Citation: Urushibara T, Hicks R (2013) Effect of Liposome Surface Charge and Peptide Side Chain Charge Density on Antimicrobial PeptideMembrane Binding as Determined by Circular Dichroism and Nuclear Magnetic Resonance Spectroscopy. J Membra Sci Technol 3: 124. doi:10.4172/2155-9589.1000124

Page 4 of 9
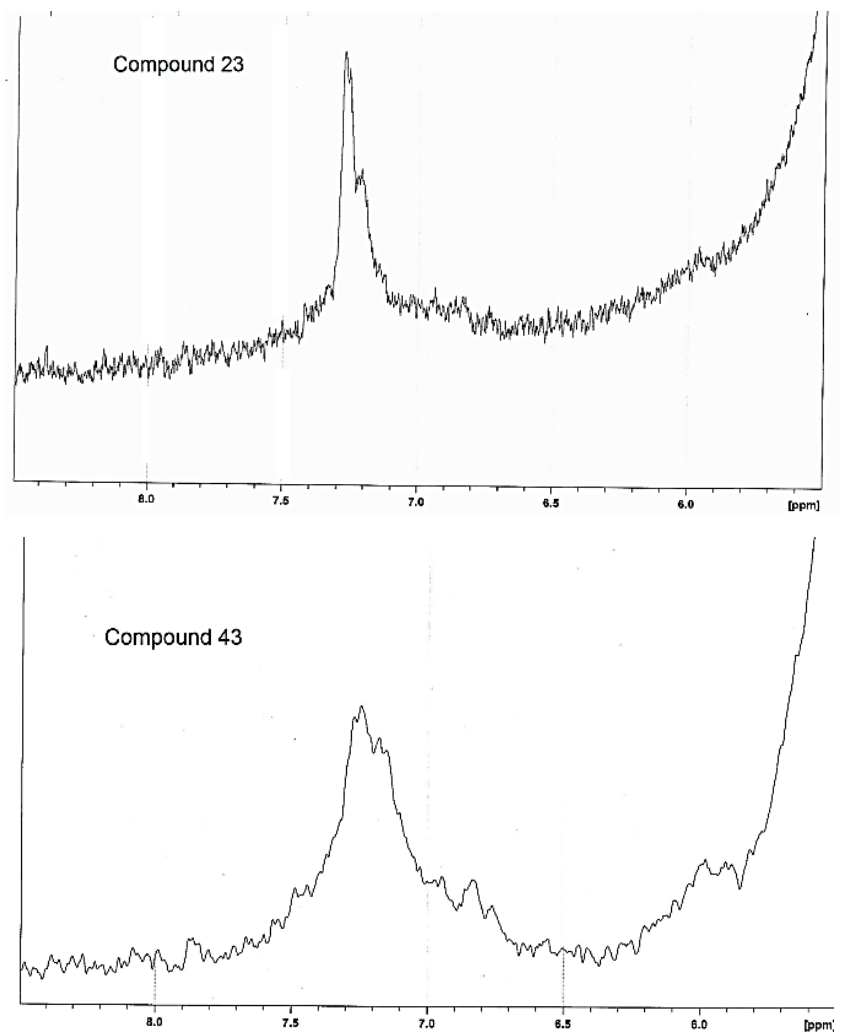

Compound 53
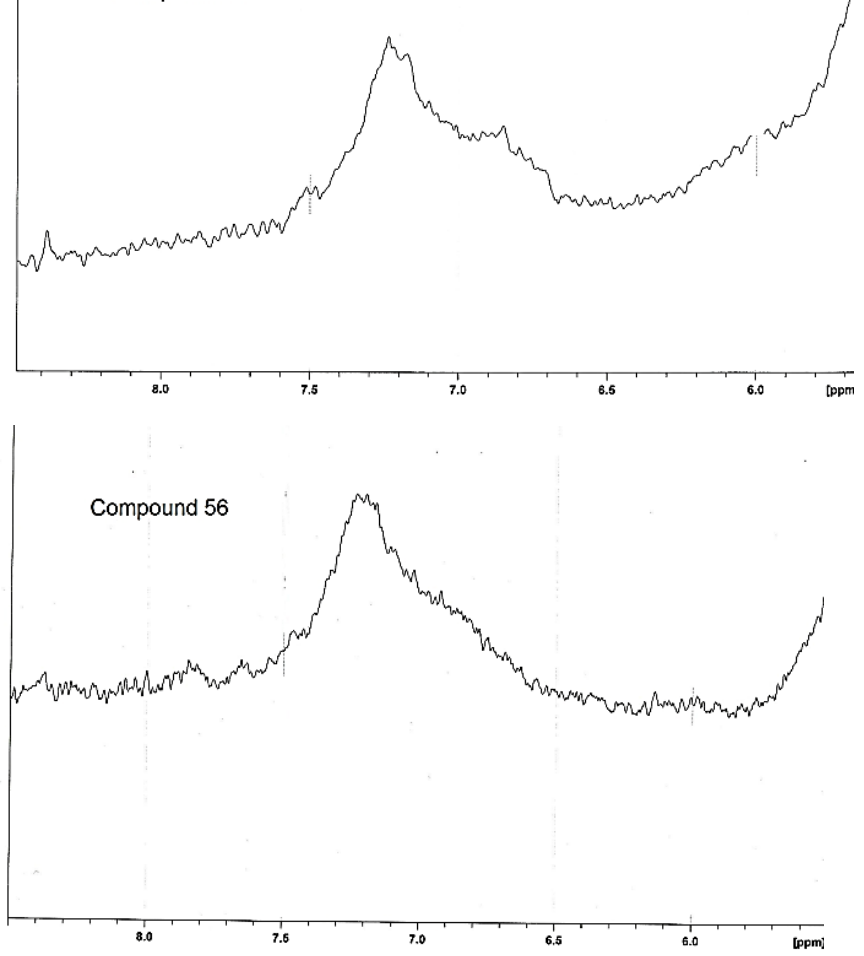

Figure 3: The aromatic region of the $400 \mathrm{MHz} 1 \mathrm{H}$ spectra of compounds 23, 43,53 , and 56 in the presence of 4:1 POPC/POPS SUVs. of the amide functionality. This proximity has the net effect of shielding the lipid surface from the full positive charge of the nitrogen atom.

\section{POPC/POPS liposomes}

The length of the side chain of the basic amino acids $(23,43,53)$ doesn't seem to effect the binding to 3:1 POPC/POPS mixed liposomes since the $\mathrm{CD}$ spectra are very similar in periodicity and shape (Figure 2). However, delocalization of the positive charge of the guanidinium group of compound 56, seems to affect the binding to $3: 1 \mathrm{POPC} /$ POPS mixed liposomes as indicated by the reduced intensity of the observed CD spectrum. Modifying the lipid composition to either 4:1 or 5:1 POPC/POPS resulted in the observation of different CD spectra for compounds, 23, 43 and 53. The shape of the spectra are similar suggesting a similar binding conformation, however the intensity is different suggesting a different binding affinity, with the CD spectrum of compound 43 being the most intense, followed by compounds 23 and 53. Once again the CD spectrum for compound 56 was the least intense. Compounds 23 and 43 exhibited very different $C D$ spectra in the presence of 3:1, 4:1 and 5:1 POPC/POPS liposomes. This observation suggests that decreasing the percentage of anionic phospholipid allows the hydrophobicity of the side chain of the basic amino acid residues to induce different binding conformations of the AMPs backbone. A possible explanation for this is at a high negative charge (3:1 POPC/ POPS) the electronic character of the liposomes dominates the AMP liposome binding interaction. As the negative charge on the liposome surface is reduced (4:1 POPC/POPS, and 5:1 POPC/POPS), the varying charge density and hydrophobic properties on the basic amino acids play a large role in defining the binding interaction of the AMP with the liposomes. Compound 56 exhibited very similar CD spectra in the presence of both 3:1 and 4:1 POPC/POPS liposomes, while compound 53 exhibited almost identical spectra in the presence of all the liposomes (Figure 2). It is interesting to point out that compound 56 with the greatest delocalization of the positive charge density exhibited the least intense CD spectra. While compound 53, with the shortest side chain of (Dab residue) exhibited the next least intense CD spectra. These two observations taken together suggest that charge delocalization plays a defining role in the binding of these AMPs to POPC/POPG SUVs.

The differences between the CD spectra of these compounds were most pronounced at POPC/POPS ratio of 4:1. The aromatic regions of the ${ }^{1} \mathrm{H}$ spectra of these four compounds were also all different, indicating different binding conformations, or mechanisms of binding for each AMP. As seen in Figure 3 the aromatic protons of compound 23appear as a narrow sharp resonance centered at approximately 7.2 $\mathrm{ppm}$. The aromatic protons of compound 43appeared as a broadened resonance again centered at approximately $7.2 \mathrm{ppm}$, a second resonance centered at approximately $6.8 \mathrm{ppm}$ was also observed. The aromatic protons of compound 53 appeared as a much broader resonance again centered at approximately $7.2 \mathrm{ppm}$, the second resonance centered at approximately $6.8 \mathrm{ppm}$ appeared broader and more intense. The aromatic protons of compound 56 appeared as a single broadened resonance from approximately 7.4 to $6.6 \mathrm{ppm}$. It is well documented in the literature that changes in the values of the chemical shifts, broadening of the resonances, or both of the aromatic protons of a Phe residue indicate the insertion of the aromatic rings into the hydrophobic region of a micelle or liposome [50,51]. Tsujii and Takagi attributed these changes to increases in the ring current effect [52]. The NMR data for the aromatic protons suggests the binding of compound 23 to the 4:1 POPC/POPS liposomes doesn't involve insertion of the aromatic rings into the liposome. However, the other three compounds show an increasing degree of insertion on the aromatic rings into the 
Citation: Urushibara T, Hicks R (2013) Effect of Liposome Surface Charge and Peptide Side Chain Charge Density on Antimicrobial PeptideMembrane Binding as Determined by Circular Dichroism and Nuclear Magnetic Resonance Spectroscopy. J Membra Sci Technol 3: 124. doi:10.4172/2155-9589.1000124

Page 5 of 9

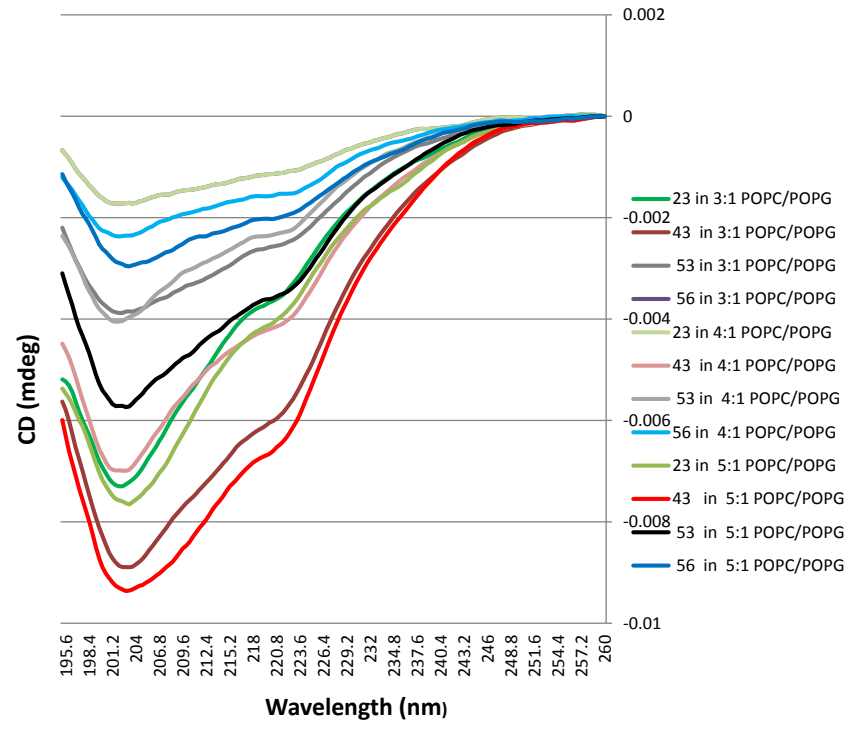

Figure 4: CD spectra of compounds 23 (green shades) 43 (red shades) 53 (black/gray shades) and compound 56 (blue shades) in the presence of 3:1 POPC/POPG SUVs, 4:1 POPC/POPG SUVs, and 5:1 POPC/POPG SUVs. The CD spectrum of 23 in 4:1 POPC/POPG SUVs is superimposed on the CD spectrum of 56 in 3:1 POPC/POPG.

hydrophobic core of the liposome in the following order $56>53>43$. This is opposite to the observed binding affinity by CD spectroscopy that indicated increasing binding in the order $43>53.56$. This suggests an alternative mechanism to the degree of insertion into the liposome is responsible for the observed broadening of the aromatic resonances. It is possible that the mechanism of binding of these AMP to this liposome is different and induces a different binding conformation onto these AMPs that results in reduced binding affinity as well as line broadening of the aromatic resonances. This hypothesis is yet to be proven.

\section{POPC/POPG liposomes}

Compounds 23, 43 and 53on binding to 3:1 POPC/POPG exhibited $\mathrm{CD}$ spectra (Figure 4) similar in shape, indicating similar binding conformations, however the intensities were different indicating different binding affinities. The intensity of the spectra increased in the order $56<53<23<43$. Binding of these compounds to $5: 1 \mathrm{POPC/}$ POPG has a minimal effect on the CD spectra of compounds 23 and 43; however the intensities of compounds 53 and 56 increased significantly.

The aromatic regions of the ${ }^{1} \mathrm{H}$ spectra of these compounds 23,43 and 53bound to 4:1 POPC/POPG SUVs are shown in Figure 5 and are all very similar indicating they adopt very similar binding conformations on interacting with 4:1 POPC/POPG liposomes. The resonances in the aromatic region are very broad indicating that the aromatic rings of both the Tic and Phe residues are inserted into the hydrophobic region of the liposome [50,51]. In addition, the aromatic protons appear as two distinct broad singlets one centered at approximately $7.75 \mathrm{ppm}$ and the second at approximately $7.15 \mathrm{ppm}$. These two resonances integrate to approximately 8 protons and 18 protons respectively. The most likely explanation for this is the resonance shifted downfield to approximately $7.5 \mathrm{ppm}$ corresponds to the 4 protons on each of the aromatic rings of Tic residues 2 and 4 . Tic residues 1 and 3 are adjacent to Phe residues which will be anchored into the hydrophobic core of the liposome; these Phe residues will also most likely cause the adjacent Tic residues to insert into the liposomes. This will result in the Tic and Phe aromatic proton exhibiting a similar chemical shifts. Tic residues number 2 and 4 are adjacent to positively charged Lys residues and thus may interact with the liposome differently than the Tic residues adjacent to Phe residues. This hypothesis has yet to be proven. The appearance of the aromatic resonances bound to 4:1 POPC/POPG liposomes are very different from those observed (Figure 3 ) on interaction with 4:1 POPC/POPS liposomes suggesting different binding conformations and a higher degree of the insertion of the aromatic rings into the hydrophobic core of the 4:1 POPC/POPG liposomes as compared to the 4:1 POPC/POPS liposomes. The CD spectra shown in Figure 6 confirm these assumptions with the $\mathrm{CD}$ spectra of compounds 23,43 and 56 observed in the presence of $4: 1 \mathrm{POPC} / \mathrm{POPG}$ being very different from those observed in the presence of 4:1 POPC/POPS. However for compound 53, the two CD spectra, are very similar. These observations
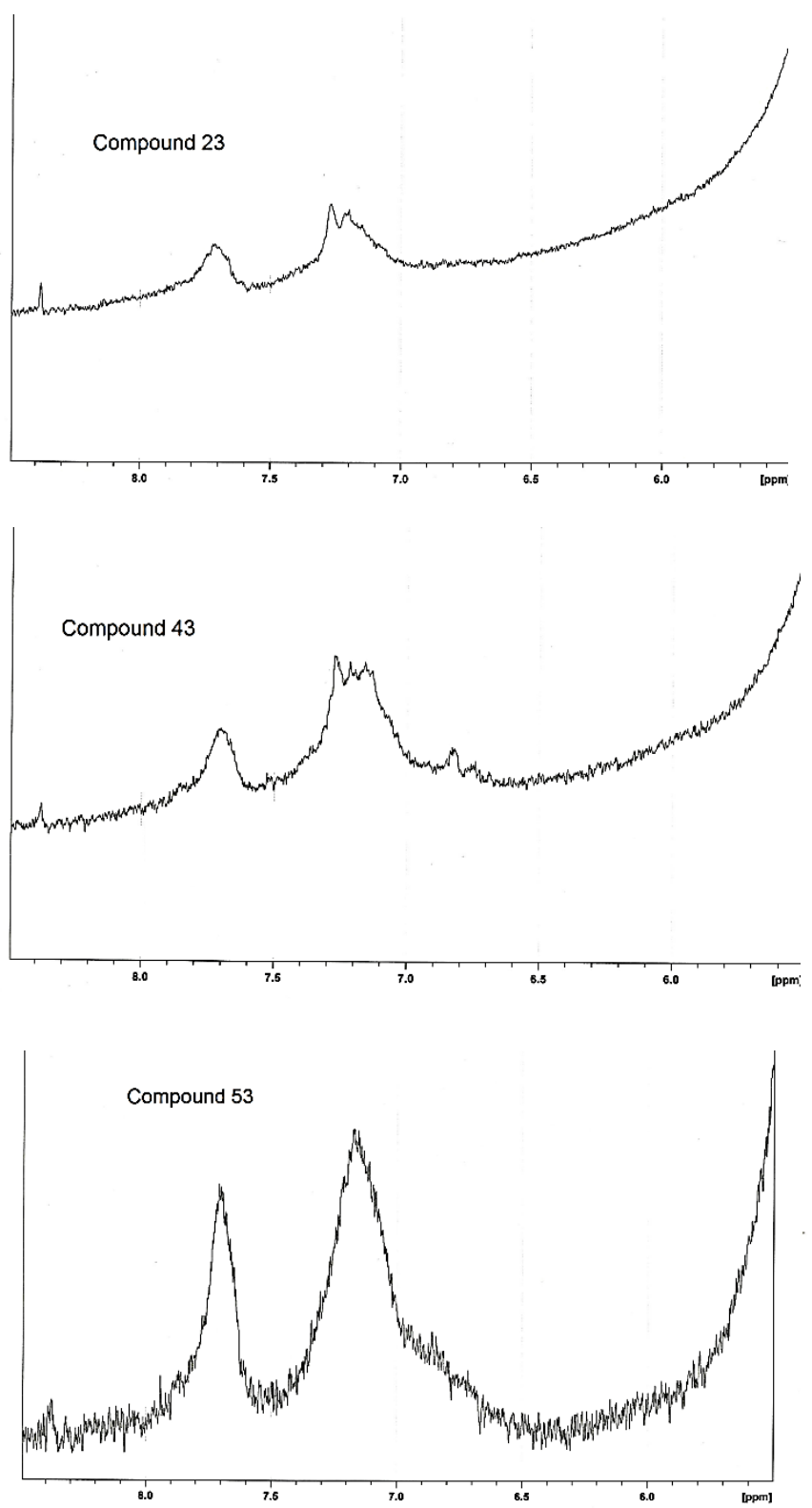

Figure 5: The aromatic region of the $400 \mathrm{MHz} 1 \mathrm{H}$ spectra of compounds 23 43 , and 53 in the presence of 4:1 POPC/POPG SUVs. 
Citation: Urushibara T, Hicks R (2013) Effect of Liposome Surface Charge and Peptide Side Chain Charge Density on Antimicrobial PeptideMembrane Binding as Determined by Circular Dichroism and Nuclear Magnetic Resonance Spectroscopy. J Membra Sci Technol 3: 124. doi:10.4172/2155-9589.1000124

Page 6 of 9

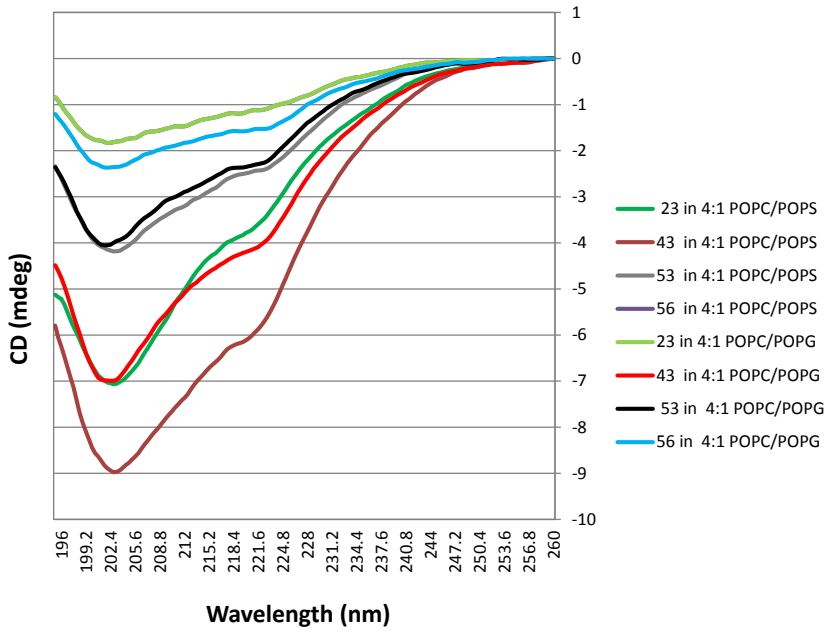

Figure 6: CD spectra of compounds 23 (green shades) 43 (red shades) 53 (black/gray shades) and compound 56 (blue shades) in the presence of $4: 1$ POPC/POPG SUVs, and 4:1 POPC/POPS SUVs. The CD spectrum of 23 in 4:1 POPC/POPG SUVs is superimposed on the CD spectrum of 56 in $3: 1$ POPC/POPS SUVs.

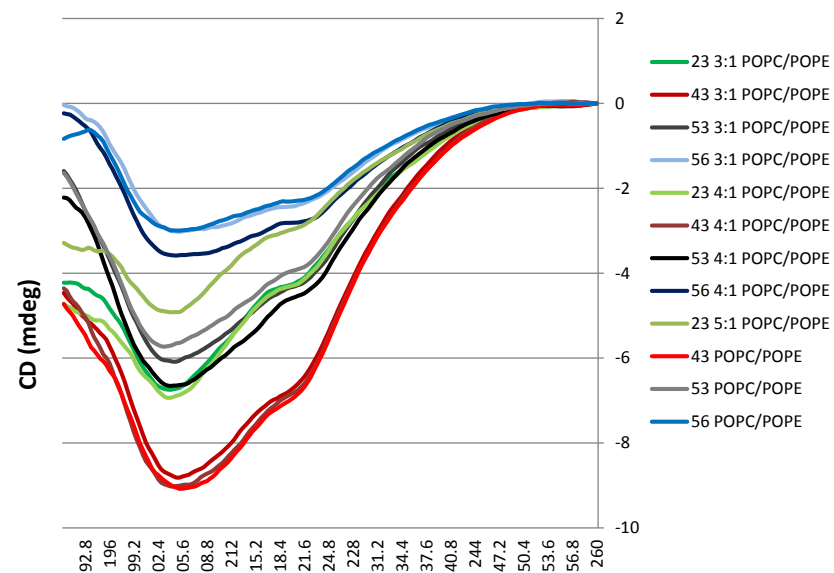

Wavelength $(\mathrm{nm})$

Figure 7: CD spectra of compounds 23 (green shades) 43 (red shades) 53 (black/gray shades) and compound 56 (blue shades) in the presence of 3:1 POPC/POPE SUVs, 4:1 POPC/POPE SUVs and 4:1 POPC/POPS SUVs.

suggest that even though the percentage of anionic lipids are the same for the 4:1 POPC/POPS and 4:1 POPC/POPG liposomes and the net charge is the same, the number of individual negative charges is different. Each POPS molecule contains one positive and two negative charges, each POPG molecule contain one negative charge. Thus the local physicochemical properties presented to the binding AMP are very different in terms of charge density and hydrophobicity.

\section{POPC/POPE liposomes}

The CD spectra of compounds $23,43,53$ and 56 in the presence of the mixed zwitterionic liposomes of 3:1, 4:1 and 5:1 POPC/POPE are different in shape and intensity indicating different binding conformations and affinities. Varying the ratios of POPC to POPE has very little effect on the $\mathrm{CD}$ spectra of the individual compounds (Figure 7) except for compound 23. This data indicates that only the Lys residues are affected by the charge in the electrostatic surface potential caused by varying the percentage of POPE incorporated into the liposome. The aromatic region of the ${ }^{1} \mathrm{H}$ NMR spectra of compounds 23,43 and 53 in the presence of 4:1 POPC/POPE are shown in Figure 8. These spectra are very similar to those observed for these compounds in the presence of sodium acetate buffer, which suggest that the aromatic rings of these peptides are not inserted into the hydrophobic core of the 4:1 POPC/ POPE liposomes. The spectra of compound 23 in the presence of 4:1 POPC/POPE liposomes is very different from that observed in pure POPC liposomes (also shown in Figure 8). The aromatic resonances of the aromatic protons of compound 23 in the presence of pure POPC liposomes are significantly broadened compared to the same resonance in the presence of 4:1 POPC/POPE liposomes. This implies that the aromatic rings of compound 23 are inserted into the hydrophobic core of the pure POPC liposomes to a much greater degree than they are in 4:1 POPC/POPE liposomes. The observed difference of the binding of this peptide to 4:1 POPC/POPE and pure POPC liposomes was unexpected. The chemical structures of POPC and POPE as shown in Figure 1 are very similar. POPC contains a quaternary ammonium salt and POPE contains a protonated primary amine. POPE therefore has a higher propensity to form intermolecular hydrogen bonds than does POPC which results in closer packing of the adjacent lipid molecules [53]. This closer packing of the lipid molecules may make it more difficult for the hydrophobic side chains of the AMP to "pass through" the hydrophilic polar head groups of the liposomes making it more difficult for the aromatic rings to insert into the hydrophobic core of the liposome. A second possible explanation for the reduced binding to 4:1 POPC/POPE liposomes compared to pure POPC liposomes is the difference in the positive charge character of the two amines. The nitrogen atom of POPC is bonded to three methyl groups, while the nitrogen atom of POPE is bonded to three hydrogen atoms. The methyl groups are of course larger than the hydrogen atoms and due to steric bulk prevent the AMP from approaching the nitrogen atom as closely as is the case with the three hydrogen atoms of POPE. Thus there may be less electrostatic repulsion between the positively charged nitrogen atom of POPC and the positively charged side chain functional groups on the AMP than that observed with POPE.

The hypothesis that these AMPs don't strongly bind to 4:1 POPC/ POPE liposomes is supported by the DOSY (Diffusion Ordered Spectroscopy) NMR data obtained for these four AMPs in the presence of 4:1 POPC/POPE liposomes. Pulse field gradient NMR methods such as the DOSY [41,54] experiment can be used to evaluate the binding affinity of peptides to small unilamellar vesicles (SUVs) [42,5557]. Since the binding of a peptide to a SUVs effectively changes the molecular size of the peptide, binding interactions can be monitored by observing changes in the peptide's diffusion coefficient [40-42,55,5865]. When interpreting diffusion data it must be remembered that the binding of a peptide to the surface of a SUVs is a steady state equilibrium process between the SUV-bound peptide and the monomeric species in solution and the observed diffusion coefficient is the weight average of the two species $[42,50,55-57,59,60,65,66]$. This equilibrium process is fast on the NMR time scale $[59,60]$. One major advantage of the DOSY experiment is that it determines simultaneously the diffusion coefficient of both the SUV and the peptide. This facilitates the detection of any peptide induced changes in the diffusion coefficient of the SUV [65].

The diffusion coefficient of compound 23 in 80 mMdeuterated sodium acetate buffer $\mathrm{pH}=6.80$ was determined using the DOSY experiment to be $1.37 \times 10^{-10} \mathrm{~m}^{2} / \mathrm{s}$. The diffusion coefficient of 23 in the presence of $28.3 \mathrm{mM} 4: 1 \mathrm{POPC} / \mathrm{POPE}$ liposomes increased to $1.74 \times 10$ ${ }^{10} \mathrm{~m}^{2} / \mathrm{s}$ while the diffusion coefficient of the $28.3 \mathrm{mM} \mathrm{4:1} \mathrm{POPC/}$ 
Citation: Urushibara T, Hicks R (2013) Effect of Liposome Surface Charge and Peptide Side Chain Charge Density on Antimicrobial PeptideMembrane Binding as Determined by Circular Dichroism and Nuclear Magnetic Resonance Spectroscopy. J Membra Sci Technol 3: 124. doi:10.4172/2155-9589.1000124

Page 7 of 9
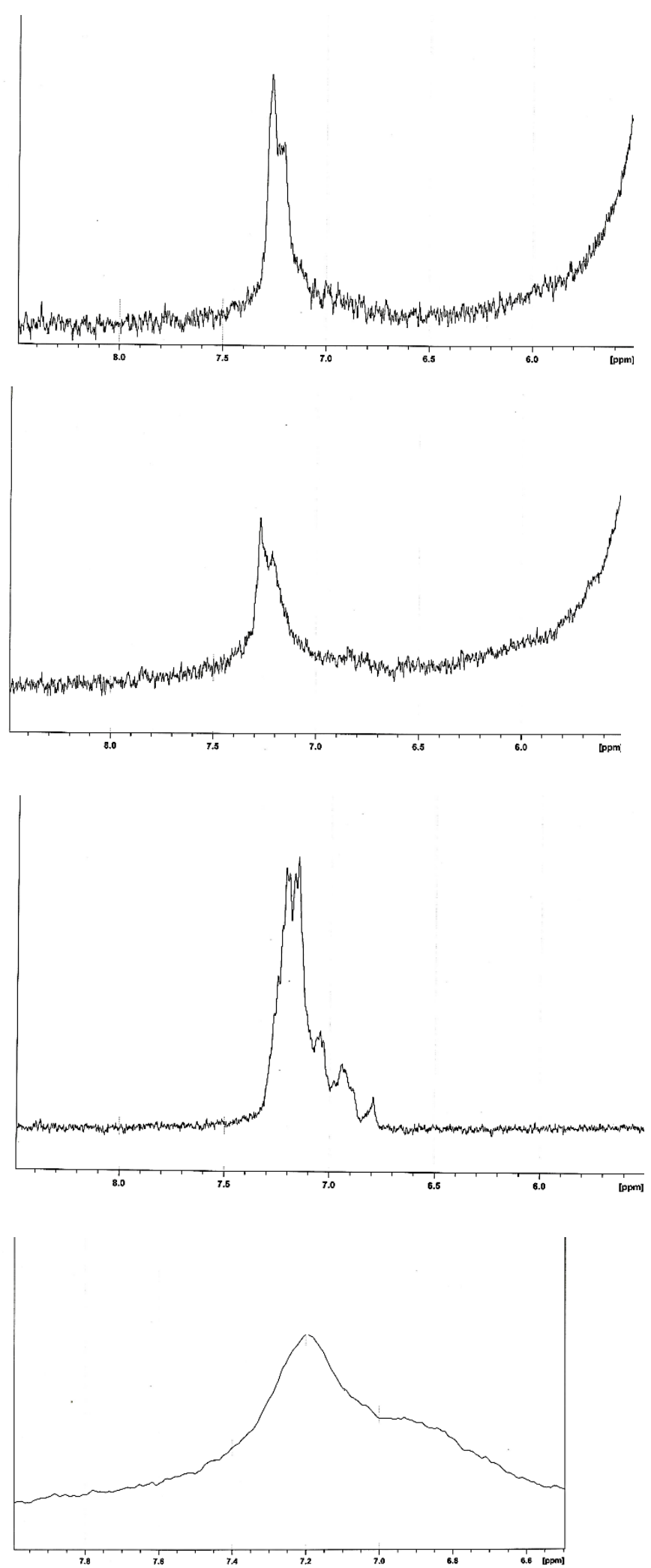

Figure 8: The aromatic region of the $400 \mathrm{MHz} 1 \mathrm{H}$ spectra of compound 23, in the presence of TOP) 3:1 POPC/POPE SUVs, SECOND) 4:1 POPC/POPE, THIRD) 5:1 POPC/POPE SUVs, BOTTOM) pure POPC SUVs.
POPE liposomes is $1.45 \times 10^{-11} \mathrm{~m}^{2} / \mathrm{s}$. This observation is surprising, if compound 23 was binding to and residing for any period of time on the surface of the $4: 1$ POPC/POPE liposomes one would expect the diffusion coefficient to of 23 to approach that of the liposome [67]. Certainly, the diffusion coefficient of compound 23 in the presence of 4:1 POPC/POPE liposomes should be slower and not faster than the diffusion coefficient in buffer [57]. As previous stated, ${ }^{1} \mathrm{H}$ NMR data clearly indicates that aromatic rings of compound 23 are not inserted into the hydrophobic core of the liposome which is consistent with the observed DOSY data. How can the increase in the diffusion coefficient be explained? The common assumption is that the diffusion coefficient of a molecule is directly related to its' molecular weight. The diffusion coefficient is related to the hydrodynamic volume of the molecule not its molecular weight $[66,67]$. The interpretation of changes in the diffusion coefficient of a molecule must take into consideration changes in molecular volume that result from major conformational changes (changes from a globular to helical structure for example) in the molecule. Therefore, the most likely explanation for the increase in the diffusion coefficient of compound 23 in the presence of 4:1 POPC/ POPE liposomes is that the electrostatic interactions that occur between the AMP and the liposome induces a conformational change onto the AMP which results in the reduction of the hydrodynamic volume of the AMP. This reduced hydrodynamic volume could manifest itself as an increase in the observed diffusion coefficient.

Attempts to determine the diffusion coefficients of $1 \mathrm{mg}$ samples of compound 23, 43, 53 and 56 in the presence of $28.3 \mathrm{mM} \mathrm{4:1} \mathrm{POPC/}$ POPG or POPC/POPS liposomes failed due to severe broadening of the aromatic proton resonances and the resulting low signal to noise ratios of those signals. Increasing the AMP concentration was not possible because even at this concentration the structure of the liposomes (as indicated by a clouding of the solution) was being changed by the binding and insertion of the AMPs into the liposomes. The two-dimensional representation of the DOSY data for a pure 4:1 POPC/POPG liposome is shown in Figure 9. All of the individual resonances are sharp and well aligned along a straight line, indicating a homogeneous liposome. In Figure 10, the two-dimensional representation of compound 53 in the presence of 4:1 POPC/POPG the resonances are broadened along the straight line. The diffusion coefficient of the liposome is increased

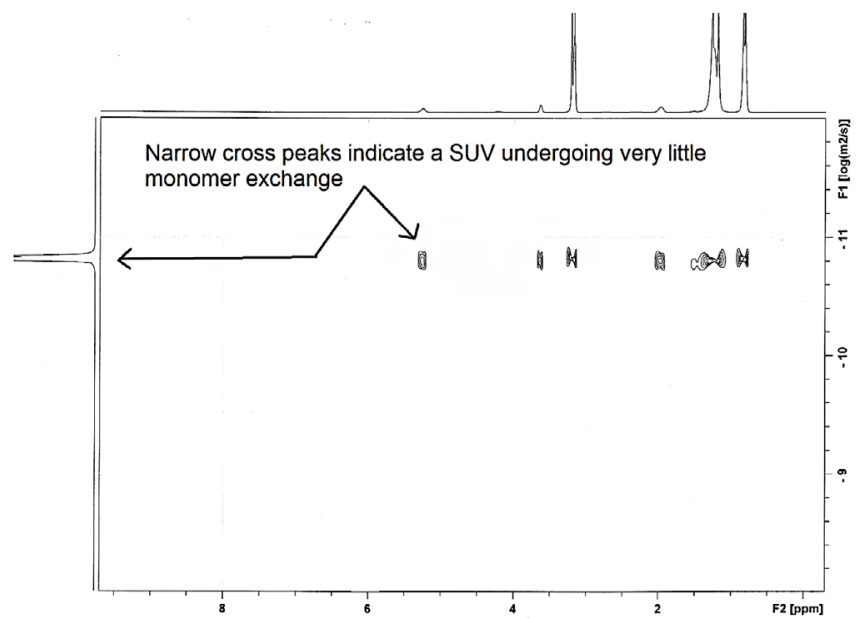

Figure 9: 2D-DOSY spectrum of 4:1 POPC/POPG SUVs. The single peak on the $y$-axis and the very sharp cross peaks also on the $x$-axis indicate that the exchange rate between the SUV bound phospholipids and the monomeric form of the phospholipids is very low. 
Citation: Urushibara T, Hicks R (2013) Effect of Liposome Surface Charge and Peptide Side Chain Charge Density on Antimicrobial PeptideMembrane Binding as Determined by Circular Dichroism and Nuclear Magnetic Resonance Spectroscopy. J Membra Sci Technol 3: 124. doi:10.4172/2155-9589.1000124

Page 8 of 9

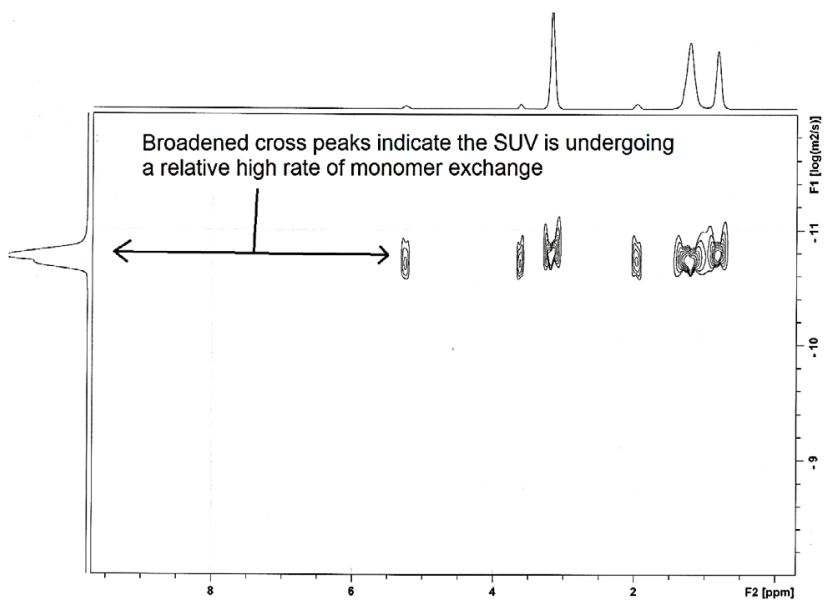

Figure 10: 2D-DOSY spectrum of 4:1 POPC/POPG SUVs and $1 \mathrm{mg}$ of compound 53. The broad peak on the $y$-axis and the broad cross peaks also on the $x$-axis indicate that the exchange rate between the SUV bound phospholipids and the monomeric form of the phospholipids is high. This indicates that the binding of the AMP to the SUV is disrupting the structure of the liposome.

to $1.51 \times 10^{-11} \mathrm{~m}^{2} / \mathrm{s}$ from $1.45 \times 10^{-11} \mathrm{~m}^{2} / \mathrm{s}$ indicating in this case a release of individual POPC or POPG molecules. As can be seen in Figure 10 cross peaks are broader than those observed in Figure 9 for the pure 4:1 POPC/POPG SUVs. This indicates that the exchange rate between the SUV bound phospholipids and the monomeric form of the phospholipids is increased. This indicates that the binding of the AMP to the SUV is disrupting the structure of the liposome.

\section{Conclusions}

This investigation demonstrates that the physicochemical properties, in this case the electrostatic surface potential and hydrophobic character of the liposome are critical in defining the binding interactions that occur between a liposome and an AMP. The previous assumption that two liposomes consisting of different phospholipids, as long as the overall charge on the liposome surface is the same, will interact in a very similar fashion with an AMP is not valid. The observation of the much weaker binding of compound 23 and 43 to mixed POPC/ POPE liposomes as compared to pure POPC liposomes is consistent with the observed antibacterial activity of these compounds, These compounds exhibited reduced activity against Gram negative bacteria which contain a high percentage of POPE such as Klebsiellapnemoniae and Pseudomonas aeruginosa [29]. This investigation has demonstrated in order to obtain biologically meaningful data concerning AMP membrane interactions using physical chemical techniques it is critical to use a liposome with a phospholipid composition as close to the target bacteria as possible. This is particularly true in the case of Gram negative bacteria containing a high percentage of POPE.

\section{References}

1. Shlaes DM, Projan SJ, Edwards JE (2004) Antibiotic discovery: state of the state. ASM News 70: 275-281.

2. Kamysz W (2005) Are antimicrobial peptides an alternative for conventional antibiotics. Nucl Med Rev 8: 78-86.

3. Zhang L, Harris SC, Falla TJ (2005) Therapeutic application of innate immunity peptides. Horizon Bioscience, San Diego, USA.

4. Toke $O$ (2005) Antimicrobial peptides: new candidates in the fight against bacterial infections. Biopolymers 80: 717-735.
5. Godballe T, Nilsson LL, Petersen PD, Jenssen H (2011) Antimicrobial b-peptides and a-peptoids. Chem Biol Drug Des 77: 107-116.

6. Ryge TS, Frimodt-Moller N, Hansen PR (2008) Antimicrobial activities of twenty lysine-peptoid hybrids against clinically relevant bacteria and fungi. Chemotherapy 54: 152-156.

7. Hancock REW (1998) The therapeutic potential of cationic peptides. Exp. Opin Invest. Drugs 7: 167-174.

8. Zasloff M (2002) Antimicrobial peptides of multicellular organisms. Nature 415 389-395.

9. Zhang L, Falla TJ (2009) Host defense peptides for use as potential therapeutics. Curr Opin Investig Drugs 10: 164-171.

10. Ganz T (2003) Defensins: antimicrobial peptides of innate immunity. Nat Rev Immunol 3: 710-720.

11. Simmaco M, Mignogna G, Barra D (1999) Antimicrobial peptides from amphibian skin: what do they tell us? Biopolymers 47: 435-450.

12. Phoenix DA, Harris F, Dennison S, Chatfield KK, Sayed Z, et al. (2003) Antimicrobial therapy: old problems - new solutions. JEC Qual L 1: 44-61.

13. Dennison SR, Wallace J, Harris F, Phoenix DA (2005) Amphiphilic a-helical antimicrobial peptides and their structure/function relationships. Protein and Peptide Letters 12: 31-39.

14. Yeaman MR, Yount NY (2003) Mechanisms of antimicrobial pepitde action and resistance. Pharmacol Rev 55: 27-55.

15. Fernandez DI, Gehman JD, Separovic F (2009) Membrane interactions of antimicrobial peptides from Australian frogs. Biochemica et Biophysica Acta 1788: 1630-1638.

16. Papo N, Shai $Y(2003)$ New lytic peptides based on the $D, L$ amphipathic helix motif preferentially kill tumor cells compared to normal cells. Biochemistry 42 : 9346-9354.

17. Findlay B, Zhanel GG, Schweizer F (2010) Cationic amphipiles, a new generation of antimicrobials inspired by natural antimicrobial peptide scaffold. Antimicrob Agents and Chemother 54: 4049-4058.

18. Huang Y, Huang J, Chen Y (2010) Alpha-helical cationic antimicrobial peptides: relationships of structure and function. Protein Cell 1: 143-152.

19. Leontiadou H, Mark AE, Marrink SJ (2006) Antimicrobial Peptides in Action. J Am Chem Soc 128: 12156-12161.

20. Hancock REW, Lehrer R (1998) Cationic peptides: a new source of antibiotics Trends Biotechno 116: 82-88.

21. Bechinger $B$ (2011) Insights into the mechanism of action of host defense peptides from biophysical and structural investigations. J Pept Sci 17: 306-314.

22. Lohner K (2009) New strategies for novel antibiotics: peptides targeting bacterial cell membranes. Gen Physiol Biophys 28: 105-116.

23. Wenk MR, Seelig J (1998) Magainin 2 amide interaction with lipid membranes: calorimetric detection of peptide binding and pore formation. Biochemistry 37 3909-3916.

24. Powers JP, Hancock RE (2003) The relationship between peptide structure and antibacterial activity. Peptides 24: 1681-1691.

25. Lohner K, Prenner EJ (1999) Differential scanning calorimetry and X-ray diffraction studies of the specificity of the interaction of antimicrobial peptides with membrane-mimetic systems. Biochim Biophys Acta 1462: 141-156.

26. Epand RM, Epand RF (2011) Bacterial membrane lipids in the action of antimicrobial agents. J Pept Sci 17: 298-305.

27. Hicks RP, Bhonsle JB, Venugopal D, Koser BW, Magill AJ (2007) De novo design of selective antibiotic peptides by incorporation of unnatural amino acids. J Med Chem 50: 3026-3036.

28. Venugopal D, klapper D, Srouji A, Bhonsle JB, Borschel R, et al. (2010) Novel antimicrobial peptides that exhibit activity against select agents and other drug resistant bacteria. Bioorg Med Chem 18: 5137-5147.

29. Hicks RP, Abercrombie JJ, Wong RK, Leung KP (2013) Antimicrobial peptides containing unnatural amino acid exhibit potent bactericidal activity against ESKAPE pathogens. Bioorg Med Chem 21: 205-214.

30. Grant GA (2002) Synthetic Peptides: A user's guide. (2nd edn), Oxford University Press, New York, NY, USA. 
Citation: Urushibara T, Hicks R (2013) Effect of Liposome Surface Charge and Peptide Side Chain Charge Density on Antimicrobial PeptideMembrane Binding as Determined by Circular Dichroism and Nuclear Magnetic Resonance Spectroscopy. J Membra Sci Technol 3: 124. doi:10.4172/2155-9589.1000124

31. Benoiton NL (2006) Chemistry of Peptide Synthesis: Taylor and Francis. CRC Press, Boca-Raton, FI.

32. Russell AL, Kennedy AM, Spuches AM, Venugopal D, Bhonsle JB, et al. (2010) Spectroscopic and thermodynamic evidence for antimicrobial peptide membrane selectivity. Chem Phys Lipids 163: 488-497.

33. Wieprecht T, Apostolov O, Seelig J (2000) Binding of the antibacterial peptide magainin 2 amide to small and large unilamellar vesicles Biophys Chem 85 : 187-198.

34. Ladokhin AS, Fernández-Vidal M, White SH (2010) CD spectroscopy of peptides and proteins bound to large unilamellar vesicles. J Membr Biol 236 : 247-253.

35. Glättli A, Daura X, Seebach D, van Gunsteren WF (2002) Can one derive the conformational preference of a beta-peptide from its $\mathrm{CD}$ spectrum? J Am Chem Soc 124: 12972-12978.

36. Ladokhin AS, Selsted ME, White SH (1999) CD spectra of indolicidin antimicrobial peptides suggest turns, not polyproline helix. Biochemistry 38 : 12313-12319.

37. Huang C (1969) Studies on phosphatidylcholine vesicles. Formation and physical characteristics. Biochemistry 8: 344-352.

38. Bringezu F, Wen S, Dante S, Hauss T, Majerowicz M, Waring A (2007) The insertion of the antimicrobial peptide dicynthaurine monomer in mode membranes: thermodynamic and structural characterization. Biochemistry 46 : 5678-5686.

39. Wei SY, Wu JM, Kuo YY, Chen HL, Yip BS, et al. (2006) Solution structure of a novel tryptophan-rich peptide with bidirectional antimicrobial activity. J Bacteriol 188: $328-334$

40. Wu DH, Chen AD, Johnson CS (1995) An improved diffusion-ordered spectroscopy experiment incorporating bipolar-gradient pulses. J Magn Reson A 115: 260-264.

41. Altieri AS, Hinton DP, Byrd RA (1995) Association of biomolecular systems via pulsed field gradient NMR self-diffusion measurements. J Am Chem Soc 117: 7566-7567.

42. Gao X, Wong TC (1998) Studies of the binding and structure of adrenocorticotropin peptides in membrane mimics by NMR spectroscopy and pulsed-field gradient diffusion. Biophys J 74: 1871-1888.

43. Bhonsle JB, Venugopal D, Huddler DP, Magill AJ, Hicks RP (2007) Application of 3D-QSAR for identification of descriptors defining bioactivity of antimicrobial peptides. J Med Chem 50: 6545-6553.

44. Russell AL, Spuches AM, Williams BC, Venugopal D, Klapper D, et al. (2011) The effect of the placement and total charge of the basic amino acid clusters on antibacterial organism selectivity and potency. Bioorg Med Chem 19: 70087022 .

45. Russell AL, Kennedy AM, Spuches AM, Gibson WS, Venugopal D, et al. (2011) Determining the effect of the incorporation of unnatural amino acids into antimicrobial peptides on the interactions with zwitterionic and anionic membrane model systems. Chem Phys Lipids 164: 740-758.

46. Russell AL, Williams BC, Spuches A, Klapper D, Srouji AH, et al. (2012) The role played by charge density and proximity to the peptide backbone on the binding interactions that occur between Antimicrobial Peptides containing unnatural amino acids and zwitter ionic and anionic liposomes. Bioorg Med Chem 20: 1723-1739.

47. Goldenberg NM, Steinberg BE (2010) Surface charge: a key determinant of protein localization and function. Cancer Res 70: 1277-1280.

48. Tossi A, Sandri L, Giangaspero A (2002) New consensus hydrophobicity scale extended to non-proteinogenic amino acids. 27th European Peptide Symposium Sorrento, Napoli, Italy.

49. Orfi L, Lin M, Larive CK (1998) Measurement of SDS Micelle-Peptide Association Using (1)H NMR Chemical Shift Analysis and Pulsed-Field Gradient NMR Spectroscopy. Anal Chem 70: 1339-1345.

50. Endo T, Shimada I, Roise D, Inagaki $F$ (1989) N-Terminal half of a mitochondrial pre sequence peptide takes a helical conformation when bound to dodecylphosphocholine micelles: a proton nuclear magnetic resonance study. J Biochem 106: 396-400.

51. Tsujii K, Takagi T (1975) Proton magnetic resonance studies of the binding of an anionic surfactant with a benzene ring to a protein polypeptide with special reference to SDS-polyacrylamide gel electrophoresis. J Biochem 77: 511-519.
52. McIntosh TJ (1996) Hydration properties of lamellar and non-lamellar phases of phosphatidylcholine and phosphatidylethanolamine. Chem Phys Lipids 81: 117-131.

53. Gurtovenko AA, Vattulainen I (2008) Membrane potential and electrostatics of phospholipid bilayers with asymmetric transmembrane distribution of anionic lipids. J Phys Chem B 112: 4629-4634.

54. Gao X, Wong TC (1999) The study of the conformation and interaction of two tachykinin peptides in membrane mimicking systems by NMR spectroscopy and pulsed field gradient diffusion. Biopolymers 50: 555-568.

55. Whitehead TL, Jones LM, Hicks RP (2001) Effects of the incorporation of CHAPS into SDS micelles on neuropeptide-micelle binding: separation of the role of electrostatic interactions from hydrophobic interactions. Biopolymers 58 593-605.

56. Whitehead TL, Jones LM, Hicks RP (2004) PFG-NMR investigations of the binding of cationic neuropeptides to anionic and zwitterionic micelles. J Biomo Struct Dyn 21: 567-576.

57. Hicks RP, Nichols Cheryl P, Kordki M, Iqbal Choudhary, Atta-Ur-Rahman (2004) The Expanding Role of NMR in Rational Drug Design: In Frontier in Medicinal Chemistry-Online, Bentham Science Publications, USA 1: 237-271.

58. Stilbs P (1982) Fourier transforms NMR pulse-gradient spin-echo (pt-pgse) self-diffusion measurements of solubilization equilibria in sds solutions. Colloid Interface Sci 87: 385-394.

59. Stilbs $P$ (1987) Fourier transform pulsed-gradient spin-echo studies of molecular diffusion. Prog Nucl Magn Reson Spectrosc 19: 1-45

60. Giuseppone N, Schmitt JL, Allouche L, Lehn JM (2008) DOSY NMR experiments as a tool for the analysis of constitutional and motional dynamic processes: implementation for the driven evolution of dynamic combinatorial libraries of helical strands. Angew Chem Int Ed Engl 47: 2235-2239.

61. Gao X, Wong TC (2001) NMR studies of adrenocorticotropin hormone peptides in sodium dodecylsulfate and dodecylphosphocholine micelles: proline isomerism and interactions of the peptides with micelles. Biopolymers 58: 2032.

62. Wong TC, Gao X (1998) The temperature dependence and thermodynamic functions of partitioning of substance P peptides in dodecylphosphocholine micelles. Biopolymers 45: 395-403.

63. Andreasson B, Nordenskiöld L, Braunlin WH (1996) An NMR self-diffusion study of the interactions between spermidine and oligonucleotides. Biopolymers 38 : 505-513.

64. Deaton KR, Feyen EA, Nkulabi HJ, Morris KF (2001) Pulse-field gradient NMR studies of sodium dodecyl sulfate micelle-peptide association. Magn Reson Chem 39: 276-282.

65. Lin M, Larive CK (1995) Detection of insulin aggregates with pulsed-field gradient nuclear magnetic resonance spectroscopy. Anal Biochem 229: 214 220

66. Clark TD, Bartolotti L, Hicks RP (2013) The Application of DOSY NMR and Molecular Dynamics Simulations to Explore the Mechanism(s) of Micelle Binding of Antimicrobial Peptides containing Unnatural Amino Acids. Biopolymers 99: 548-561.

67. Jonsson BH, Wennerstrom PG, Nilsson P, Linse $P$ (1986) Self-diffusion of smal molecules in colloidal systems. Colloid Polym Sci 264: 77-88. 